\title{
A SPECTRAL MAPPING THEOREM FOR TENSOR PRODUCTS OF UNBOUNDED OPERATORS
}

\author{
BY MICHAEL C. REED ${ }^{1}$ AND BARRY SIMON ${ }^{2}$
}

Communicated by William Browder, February 4, 1972

1. Introduction. In this note we will discuss the spectrum of tensor products of not necessarily bounded operators on Banach spaces $X$ and $Y$. $X \otimes Y$ will denote the tensor product of $X$ and $Y$ in some uniform cross-norm [1]. Thus, (i) $X \otimes Y$ is the completion of the algebraic tensor product in a norm with $\|x \otimes y\|=\|x\|\|y\|$; (ii) for any $A \in \mathscr{L}(X)$, the bounded operators on $X$, and $B \in \mathscr{L}(Y)$, there is an operator $A \otimes B \in$ $\mathscr{L}(X \otimes Y)$ with $(A \otimes B)(x \otimes y)=A x \otimes B y$ and $\|A \otimes B\|=\|A\|\|B\|$. Typical examples of such uniform cross-norms are the usual Hilbert space tensor product norm and the $L^{p}$ norm on $L^{p}(X \otimes Y, d \mu \otimes d v)=L^{p}(X, d \mu)$ $\otimes L^{p}(Y, d v)(1 \leqq p<\infty)$.

Given a polynomial (or a rational function) in two variables and closed operators $A$ on $X$ and $B$ on $Y$, we want to discuss the spectrum of $P(A \otimes I, I \otimes B)$ as an operator on $X \otimes Y$. For unbounded operators, one must define what it means for an operator $C$ on $X \otimes Y$ "to be" $P(A \otimes I, I \otimes B)$. We take a fairly strong definition:

DEFINITION 1. Given a closed operator $A$ with nonempty resolvent set on a Banach space, $X$, we say that a sequence $A_{n}$ of bounded operators on $X$ is an $\mathscr{R}(A)$-approximation if and only if $A_{n}$ converges to $A$ in norm resolvent sense [2] and each $A_{n}$ is a polynomial in resolvents of $A$.

Definition 2. Given closed operators $A$ and $B$ on Banach spaces $X$ and $Y$, and a rational function, $P(z, \omega)$, we say that a closed operator $C$ on $X \otimes Y$ equals $P(A \otimes I, I \otimes B)$ (or $P(A, B)$, for short) if and only if, there exists an $\mathscr{R}(A)$-approximation, $A_{n}$, and an $\mathscr{R}(B)$-approximation, $B_{n}$, so that $P\left(A_{n}, B_{n}\right)$ converges in norm resolvent sense to $C$.

Existence and uniqueness questions for $P(A, B)$ naturally arise. In applying Theorem 1 below, all the hard analysis is in proving that existence holds. The existence and uniqueness question is discussed in detail in a forthcoming paper [3], primarily in the case where $A$ and $B$ are generators of bounded holomorphic semigroups. In the general case, we do not know whether it is possible for two different operators $C$ and $C^{\prime}$ to both "equal" $P(A, B)$ but in that case our proof of Theorem 1 implies that $(C-\lambda)^{-1}$ $-\left(C^{\prime}-\lambda\right)^{-1}$ is quasinilpotent.

AMS 1970 subject classifications. Primary 47A60; Secondary 46L15.

Key words and phrases. Spectrum, tensor products, Gelfand theory.

${ }_{1}$ On leave from the Department of Mathematics, Princeton University.

2 A Sloan Foundation Fellow. 
THEOREM 1. Let $X$ and $Y$ be Banach spaces and let $X \otimes Y$ be their tensor product in some uniform cross-norm. Let $A$ and $B$ be closed operators on $X$ and $Y$ respectively with nonempty resolvent sets so that each is either bounded or has unbounded spectra. Let $C=P(A \otimes I, I \otimes B)$ in the sense of Definition 2. Then

$$
\sigma(C)=\overline{P(\sigma(A), \sigma(B))}=\overline{\{P(\lambda, \mu) \mid \lambda \in \sigma(A), \mu \in \sigma(B)\}} \text {. }
$$

REMARKS. 1. This is the main theorem of this note. However, we feel the method of proof is sufficiently interesting in its own right, so we introduce some of the ideas below.

2. The interesting case of Theorem 1 occurs when $P(\sigma(A), \sigma(B))$ is not closed. This can only occur if at least one of the operators is unbounded. That it can occur is easily seen by choosing suitable selfadjoint operators and applying the spectral theorem.

3. Our hypothesis that $\sigma(A)$ be unbounded if $A$ is unbounded rules out such pathological operators as inverses of quasinilpotents with trivial kernel and cokernel which have empty spectra. The hypothesis will enter the proof because it will allow us to approximate "the spectrum at infinity" with the spectrum at finite points.

4. Spectra of tensor products have been discussed in [4], [5], [6]. The case of Theorem 1 where both $A$ and $B$ are bounded has already been proven by Schecter. Only Ichinose has discussed the unbounded case and he has restricted himself to cases where $P(\sigma(A), \sigma(B))$ is closed.

5. Our interest in the problem of the spectra of tensor products was aroused by recent work on $N$-body Schrödinger operators which required an analysis of the spectra of non-normal sectorial operators [7], [8].

6. Our results extend to tensor products of $n$ operators.

2. The bounded case. The key tool in the proof of Theorem 1 is the Gelfand theory of commutative Banach algebras. We first need to analyze the bounded case precisely. The algebras we will use are

Definition 3. Let $A \in \mathscr{L}(X)$. $\mathscr{R}(A)$ will be the Banach subalgebra of $\mathscr{L}(X)$ generated by $\mathscr{R}_{0}(A)$, the polynomials in resolvents of $A$.

Notice that $A \in \mathscr{R}(A)$ since $A=\lim _{\lambda \rightarrow \infty}\left[\lambda^{2}(\lambda-A)^{-1}-\lambda\right]$. Moreover since $(A-\mu)(A-\mu)^{-1}=1$ for any $\mu \in \rho(A)$, the resolvent set for $A$, $l\left((A-\mu)^{-1}\right)=(l(A)-\mu)^{-1}$ for any $l \in \sigma(\mathscr{R}(A))$, the Gelfand spectrum of $\mathscr{R}(A)$. Thus $\hat{A}$, the Gelfand transform of $A$, is a homeomorphism of $\sigma(\mathscr{R}(A))$ and $\sigma(A)$ so $\sigma(\mathscr{R}(A))$ can be thought of as a subset of $C$. This is true even though $\mathscr{R}(A)$ is not generated by $A$ if $\rho(A)$ is not connected. Notice that the spectrum of $A$ relative to $\mathscr{R}(A)$ is by construction its spectrum as an element of $\mathscr{L}(X)$. 
Given $A \in \mathscr{L}(X)$ and $B \in \mathscr{L}(Y)$, and $X \otimes Y$ in a uniform cross-norm, we let $\mathscr{R}_{0}(A) \otimes \mathscr{R}_{0}(B)$ be the algebra in $\mathscr{L}(X \otimes Y)$ of finite sums of tensor products of operators in $\mathscr{R}_{0}(A)$ and $\mathscr{R}_{0}(B)$ and we let $\mathscr{R}(A) \hat{\otimes} \mathscr{R}(B)$ be its closure in $\mathscr{L}(X \otimes Y)$. Then, when $A \in \mathscr{L}(X), B \in \mathscr{L}(Y)$ :

THEOREM 2. $\sigma(\mathscr{R}(A) \otimes \widehat{\otimes} \mathscr{R}(B)) \cong \sigma(\mathscr{R}(A)) \times \sigma(\mathscr{R}(B))$.

TheOREM 3. If $C \in \mathscr{R}_{0}(A) \otimes \mathscr{R}_{0}(B)$, in particular, if $C$ is a polynomial in $A \otimes I$ and $I \otimes B$, then

$$
\mathscr{R}(C) \subset \mathscr{R}(A) \hat{\otimes} \mathscr{R}(B) .
$$

Remarks. 1. Theorem 2 is a statement that the tensor product of $l_{1} \in \sigma(\mathscr{R}(A))$ and $l_{2} \in \sigma(\mathscr{R}(B))$ which has an obvious definition on $\mathscr{R}_{0}(A) \otimes$ $\mathscr{R}_{0}(B)$ is bounded and so extends to $\mathscr{R}(A) \hat{\otimes} \mathscr{R}(B)$. We prove this only for the tensor product of multiplicative linear functionals. By a very different method one can prove that the tensor product of arbitrary linear functionals extends [11].

2. Theorem 3 is an improvement of a result of Schechter who proved that $\mathscr{R}(C)$ is in the double commutant of $\mathscr{R}(A) \otimes \mathscr{R}(B)$.

3. In case $A$ and $B$ are bounded, Theorem 1 follows easily from Theorems 2 and 3.

4. The key lemma in the proof of Theorems 2 and 3 is that when $C \in \mathscr{R}_{0}(A) \otimes \mathscr{R}_{0}(B)$, then the spectrum of $C$ as an element of $\mathscr{L}(X \otimes Y)$ contains $\left\{\left(l_{1} \otimes l_{2}\right)(C) \mid l_{1} \in \sigma(\mathscr{R}(A)), l_{2} \in \sigma(\mathscr{R}(B))\right\}$. This is proven using the method of Brown and Pearcy [4] together with the following fact from the theory of several complex variables [9]: If $f$ is holomorphic in a neighborhood of $D$, a compact subset of $C^{2}$, and $f$ vanishes at a point in $D$, then $f$ vanishes somewhere on $\partial D$, the topological boundary of $D$.

3. Applying multiplicative linear functionals to unbounded operators. The second key element in the proof of Theorem 1 involves the application and refinement of an idea in Hille-Phillips [10].

Let $A$ be a closed unbounded operator on $X$ with nonempty resolvent set. Define $\mathscr{R}(A)$ to be the closed algebra generated by the resolvents of $A$. By the first resolvent identity, $\mathscr{R}(A)=\mathscr{R}\left((A-\mu)^{-1}\right)$ for any $\mu \in \rho(A)$.

Definition 4. Pick $\mu \in \rho(A)$. Given $l \in \sigma(\mathscr{R}(A))$, define $l(A)$ to be $\infty$ if $l\left((A-\mu)^{-1}\right)=0$ and to be $\mu+l\left((A-\mu)^{-1}\right)^{-1}$ if $l\left((A-\mu)^{-1}\right) \neq 0$.

Again by the first resolvent identity, $l(A)$ is independent of the choice of $\mu \in \rho(A)$. This definition has several important properties: first we can define $\hat{A}$ as a continuous map from $\sigma(\mathscr{R}(A))$ to the Riemann sphere and $\hat{A}$ sets up a homeomorphism between $\sigma(\mathscr{R}(A))$ and $\sigma(A) \cup\{\infty\}$; secondly, if $A_{n}$ is any $\mathscr{R}(A)$-approximation in the sense of Definition 1, then $l\left(A_{n}\right) \rightarrow$ $l(A)$ for any $l \in \sigma(\mathscr{R}(A))$. 
Theorem 1 now follows from several remarks: (a) By Definition 2 and Theorem 2, $\mathscr{R}(C) \subset \mathscr{R}(A) \hat{\otimes} \mathscr{R}(B)$ even in the unbounded case. (b) Thus $\sigma(C)$ as an element of $\mathscr{L}(X \otimes Y)$ is $\{l(C) \mid l \in \sigma(\mathscr{R}(A) \hat{\otimes} \mathscr{R}(B)), l(C) \neq \infty\}$. (c) By hypothesis, if $A$ is unbounded, $\infty$ is not an isolated point of $\sigma(A) \cup\{\infty\}$ so $\sigma(A)$ is dense in $\sigma(\mathscr{R}(A))$. (d) Thus, by Theorem $3, \sigma(A) \times \sigma(B)$ is dense in $\sigma(\mathscr{R}(A) \hat{\otimes} \mathscr{R}(B))$. (e) Finally ran $\hat{C}=\{l(c) \mid l \in \sigma(A) \times \sigma(B)\}$ since $\hat{C}$ is continuous and $\sigma(A) \times \sigma(B)$ is dense.

\section{REFERENCES}

1. R. Schatten, $A$ theory of cross-spaces, Ann. of Math. Studies, no. 26, Princeton Univ. Press, Princeton, N.J., 1950. MR 12, 186.

2. T. Kato, Perturbation theory for linear operators, Die Grundlehren der math. Wissenschaften, Band 132, Springer-Verlag, New York, 1966, pp. 206-207. MR 34 \# 3324.

3. M. Reed and B. Simon, Tensor products of closed operators on Banach spaces (to appear).

4. A. Brown and C. Pearcy, Spectra of tensor products of operators, Proc. Amer. Math. Soc. 17 (1966), 162-166.

5. M. Schecter, On the spectra of operators on tensor products, J. Functional Analysis 4 (1969), 95-99. MR 41 \#2431.

6. T. Ichinose, On the spectra of tensor products of linear operators in Banach spaces, J. Reine Angew. Math. 244 (1970), 119-153; Operators on tensor products of Banach spaces (to appear); Operational calculus for tensor products of linear operators in Banach spaces (to appear).

7. E. Balslev and J. M. Combes, Spectral properties of many-body Schrödinger operators with dilatation-analytic interactions, Comm. Math. Phys. 22 (1971), 280-294.

8. B. Simon, Resonances for dilatation analytic potentials and the foundations of time dependent perturbation theory (to appear).

9. R. Gunning and B. Rossi, Analytic functions of several complex variables, PrenticeHall Series in Modern Analysis, Prentice-Hall, Englewood Cliffs, N.J., 1965, p. 106. MR 31 \# 4927; R. Nachbin, Holomorphic functions, domains of holomorphy, and local properties, North-Holland, Amsterdam, 1970, p. 43.

10. E. Hille and R. S. Phillips, Functional analysis and semi-groups, rev. ed., Amer. Math. Soc. Colloq. Publ., vol. 31, Amer. Math. Soc., Providence, R.I., 1957. MR 19, 664.

11. B. Simon, Uniform cross-norms (to appear). 48104

Department of Mathematics, University of Michigan, Ann Arbor, Michigan

Department of Mathematics, Princeton University, Princeton, New Jersey 08540

Current address: (Michael C. Reed) DePartment of Mathematics, Princeton UniverSITY, PRINCETON, NeW JERSEY 08540 